\title{
The relationship between cognitive, motor-kinesthetic, and oculomotor adaptation'
}

\author{
ROBERT G. WEBSTFR 2 \\ SANDIA LABORATORIES. ALBLQUERQUE. NEW UEXIC')
}

\begin{abstract}
The literature concerning adaptation to prism indicates that several adaptive mechanisms may be important. The particular mechanism or mechanisms involved depends (at least in part) upon the type of adaptive exposure. In the present study. three adaptive mechanisms (cognitive, oculomotor, and motor-kinesthetic / were investigated. Ss were asked to point in the dark at an illuminated target. The target was seen displaced from its veridical position due to a wedge prism placed before S's right eye. The left eye was occluded. Ss then viewed their visual target pointing errors through the displacing prism without seeing any part of their bodies. One group of Ss was instructed to ignore these prism-induced errors and to continue pointing at the target's visual position. A second group of $S_{s}$ was instructed to compensate fully for their errors and to attempt to eliminate them on all future trials. For the latter group errors were completely eliminated, while for Ss instructed to ignore their errors, relatively small improvement in visual target settings occurred. This improvement was called cognitive adaptation, since it depended on the $S$ 's conscious control. In addition. for both conditions. evidence was found that allowing $S s$ to view their prism-induced pointing errors resulted in some form of motor-kinesthetic adaptation. This adaptation was hypothesized to represent a change in the judged position of the pointing hand relative to its felt position. It was concluded that this motor-kinesthetic adaptation was dependent, in part, upon cognitive information concerning the effects of the prism and that it serves to reduce conflict between cognitive and visual cues, i.e., between what $S$ believes and what he sees.
\end{abstract}

Helmholtz ${ }^{3}$ in 1867 was among the first investigators to note the phenomenon of prism adaptation. With base right wedge prisms placed before his eyes he reported that "objects in the field of view will all apparently be shifted to the left of their real position." When, with eyes closed, Helmholtz tried to touch some object after viewing it through prisms, he normally missed. However, when, after several attempts at striking the object with or without opening his eyes, Helmholtz again viewed the object, he was able to reach correctly when he was retested with eyes closed. Helmholtz found that this adaptation transferred fully to his nonreaching hand and concluded that this acquired bimanual accuracy was due not to a change in "muscular feeling of the hand ... or the judgment of its position" but rather to the change in "judgment of the direction of gaze."

Harris (1963) described a different locus for the adaptive effect. Harris's Ss pointed repeatedly at a target while viewing their pointing hand through a wedge prism. Instead of transferring intermanually, as in Helmholtz's experiment, adaptation was confined to the pointing hand. Harris concluded that prism adaptation may be a proprioceptive phenomenon involving a change in the felt position of the exposed hand.

Recently, McLaughlin and his associates have demonstrated that both these adaptive mechanisms are important and may operate concurrently. McLaughlin and Rifkin (1965) had Ss in the dark point straight ahead and at an illuminated visual target viewed through a displacing prism (hand not seen). Ss were then asked to correct any visual target pointing errors.
Fvidence was tound for two adaptive mechanisms: Helmholtz' change in the judgment of the direction of gaze and Harris's proprioceptive change.

McLaughlin, Rifkin. and Webster (1966) found that adaptation to prism may occur even when $S$ is unable to see either his prism-induced pointing errors or any part of his body through the prism. This adaptation involved an apparent change in the judgment of the direction of gaze. This oculomotor adaptation was thought to be calused by two secondary prismatic effects. (a) asymmetry of. the visual display and (b) apparent rotation of frontai-parallel surfaces about their vertical axes.

McLaughlin and Webster (1967) have obtained direct measurement of changes in eye position resulting during adaptation to prism. These changes were shown to be directly related to changes in S's pointer settings. Evidence for concurrent proprioceptive adaptation was also obtained.

In addition to oculomotor and proprioceptive adaptation a third type is possible. This form of adaptation, which will be called cognitive, depends upon feedback to Ss concerning the nature of their prism-induced pointing errors. Weinstein. Sersen, Fisher, and Weisinger (1964) had Ss while wearing displacing prisms orient themselves so that a veridically straight-ahead target bisected their body midline. Following each response any error was corrected thus indicating to $S$ the direction and magnitude of his error. Comparison of pre- and postexposure settings made without prisms revealed significant adaptive aftereffects.

Similarly, Howard, Craske, and Templeton (1965) report on the role of cognitive cues in adaptation to optical displacement. A rod seen displaced by mirrors was moved head-on toward S. Significant adaptive aftereffects in pointing at visual targets resulted only for Ss who were hit on the lips by the rod. No aftereffects occurred if the rod was moved toward $\mathrm{S}$ but failed to touch him.

\section{METHOD}

In the present experiment cognitive adaptation was studied and the relationship between it and proprioceptive and oculomotor adaptation was investigated. Eye position was monitored during the course of adaptation to give a measure of oculomotor adaptation. Measurements of pointing behavior were also taken at frequent intervals before, during, and after adaptive exposure to determine the amount of proprioceptive and cognitive adaptation.

Subjects

The Ss were 24 right-handed male college and high school students who were paid for their services.

\section{Apparatus}

The visual display consisted of a horizontal row of dots located $5.5 \mathrm{~cm}$ below eye level on a black vertical partition perpendicular to S's line of sight. The dots (34 on each side of the center dot) were $3 \mathrm{~mm}$ in diam and were spaced, on center, at $1-\mathrm{cm}$ intervals. These dots were visible either when transilluminated or when illuminated indirectly by two lights located on the sides of the apparatus. The center dot could be transilluminated independently of the other dots. The $S$ 
viewed this display monocularly from a distance of approximately $37 \mathrm{~cm}$, his head being held in position by a bite board, chin rest, and forehead rest. When making the dental impression, $\mathbf{S}$ was instructed to position himself so that the center dot (viewed binocularly) appeared to be straight ahead with respect to his head and body.

The S's left eye was occluded throughout the experiment (except during the making of the bite board). The prism ( 20 diopter base right) was positioned in front of S's right eye by a remote control system. Positioning of the prism occurred in the dark. Thus, $\mathrm{S}$ started the experiment without having seen the visual display through the prism.

The right eye was photographed through the prism and through an aperture placed in the vertical partition containing the visual display. The aperture was camouflaged to prevent $S$ from using it as a cue to straight ahead. A $35-\mathrm{mm}$ camera was positioned with its focal plane $75 \mathrm{~cm}$ from the eye. The eye was imaged on the film (Kodak High-Speed Infrared) by a telephoto lens having a focal length of $18 \mathrm{~cm}$ and positioned with its center approximately $46 \mathrm{~cm}$ from the eye. A small projector provided infrared illumination for photography and was directed at $S$ 's right eye from the right side. Light from this projector was not visible to $S$ during the experiment.

Located $5 \mathrm{~cm}$ below the row of dots was a sliding pointer which $S$ could move along a horizontal track perpendicular to his line of sight. The pointer was $3 \mathrm{~cm}$ wide and had a tactual reference mark at its midpoint. The position of S's pointer was indicated to $E$ on a centimeter scale by means of a second pointer, integral with the first, located on E's side of the apparatus. The $\mathrm{E}$ was able to change the position of S's pointer.

An opaque partition was placed in the horizontal plane just below the row of dots. This partition prevented $S$ from seeing his hands or any part of his body. The tip of S's pointer was visible above the partition. The $S$ could see the pointer tip only if $E$ illuminated the visual display by means of two "side lights."

Also installed in the vertical partition directly straight ahead of $S$ and $15 \mathrm{~cm}$ above the row of dots was a small 8-ohm speaker, $6 \mathrm{~cm}$ in diam. A tape recorder, set to play intermittent white noise ( $0.5 \mathrm{sec}$ on- $0.5 \mathrm{sec}$ off), was connected to the speaker. The opening for the speaker was not visible to $S$.

\section{Procedure}

Prior to the experiment, $\mathrm{S}$ was positioned on the bite board and was shown how to operate the pointer. The projector used to illuminate the right eye was positioned and the camera was focused. All lights were extinguished and the prism was positioned in front of S's right eye.

The sequence of events in the experiment itself for all Ss was as follows: Twelve preexposure pointer settings, 10 adaptive exposure trials, and 12 postexposure settings. For each of the 12 preexposure settings $E$ placed the pointer $8-12 \mathrm{~cm}$ toward S's right or left. The $S$ was then told which side the pointer was on and was instructed to take hold of it with his right (or left) hand and to make a setting. Three types of pointer settings were made: Straight ahead, visual target, and auditory target. The $S$ was instructed that all straight-ahead settings were to be made with reference to his head and body not his right eye. Prior to each visual target setting, the center dot was transilluminated and $S$ was instructed to set the pointer under the dot. The apparent visual position of the center dot was displaced $6.6 \mathrm{~cm}$ to the left of center by the prism. Prior to each auditory target setting the intermittent white noise was turned on and played until $S$ had finished setting the pointer under the speaker. Completion of each of the 12 pointer settings was signaled by $S$ who tapped his finger on the table. The order of presentation of settings was balanced within and across Ss for type of setting, hand used, and pointer starting position.

Following the 12 preexposure settings, the row of dots was illuminated and 10 adaptive exposure trials were conducted. Only the right hand was used to make pointer settings during these 10 trials; prior to each setting the pointer was positioned $8-12 \mathrm{~cm}$ to the left or right of center. Each exposure trial began with $S$ being asked to set the pointer straight ahead of the midline of his body. Following this setting $S$ was asked to look straight ahead and a photograph was taken. When looking straight ahead $S$ was told to fixate one of the dots or a point between two illuminated dots. (This was to ensure that $S$ was looking in the horizontal plane when the picture was taken.) Following the photograph, illumination of the dots was extinguished for about $3 \mathrm{sec}$. The center dot alone was then transilluminated and $S$ was asked to make a visual target pointer setting.

Completion of the two pointer settings and a photograph was followed by $5 \mathrm{sec}$ of adaptive exposure during which the side lights were turned on allowing $S$ to see any error in his visual target pointer setting. After allowing $S$ to note his error $E$ moved the pointer back and forth a total of $40 \mathrm{~cm}(10 \mathrm{~cm}$ to the left and right of the pointer's starting position) instructing $S$ to watch the moving pointer and then returned the pointer to its original position. Following this adaptive exposure all lights were turned off, the row of dots was again illuminated, and a new trial was begun.

Upon completion of the 10 exposure trials, 12 postexposure pointer settings were made by $S$. These settings were identical to the preexposure settings. The order of presentation of settings given to any one $S$ was identical for both the pre- and postexposure periods.

Two experimental groups of Ss were given the above procedure, each group consisting of 12 Ss. Ss in Group I were instructed to ignore any errors in their visual target pointer settings and to continue to "set the pointer where you see the bright dot."

The Group 2 Ss were instructed to utilize information gained from viewing their errors and to attempt to eliminate these errors on future trials. This emphasis on making visual target settings on the basis of apparent physical (i.e., veridical) position rather than on apparent visual position was placed on all visual target settings made before, during, and after the 10 exposure trials. Thus, Ss were instructed to "set the pointer beneath the bright dot."

\section{Reliability of Auditory Target Settings}

Upon completion of the postexposure settings, eight additional auditory target settings were made. If the standard deviation of these settings exceeded $4.0 \mathrm{~cm}$ for any $S$, his results were excluded from further analysis. Three Ss (out of an original group of 27) were eliminated in this fashion. This procedure was necessary because some $\mathrm{Ss}$ were unable to make auditory target settings reliably.

\section{Calibration Photos}

Following the eight auditory target settings and with the prism still in place and the side lights on, E placed S's pointer beneath the dot, $8 \mathrm{~cm}$ toward S's right from the center dot. The $S$ was instructed to fixate the dot above the pointer and when $S$ signaled that he was ready a photo was taken. This procedure was continued with $S$ fixating, in turn, the dots at $6,4,2,0$, and $-2 \mathrm{~cm}$ to the right of center. This sequence of six photos was repeated three or four times depending upon amount of film available.

\section{Measurement of Eye Position}

The $35-\mathrm{mm}$ infrared transparencies were projected to give an overall magnification of about 30 . The distance $d$ from an anatomical mark on the outer canthus to the nearest point on 


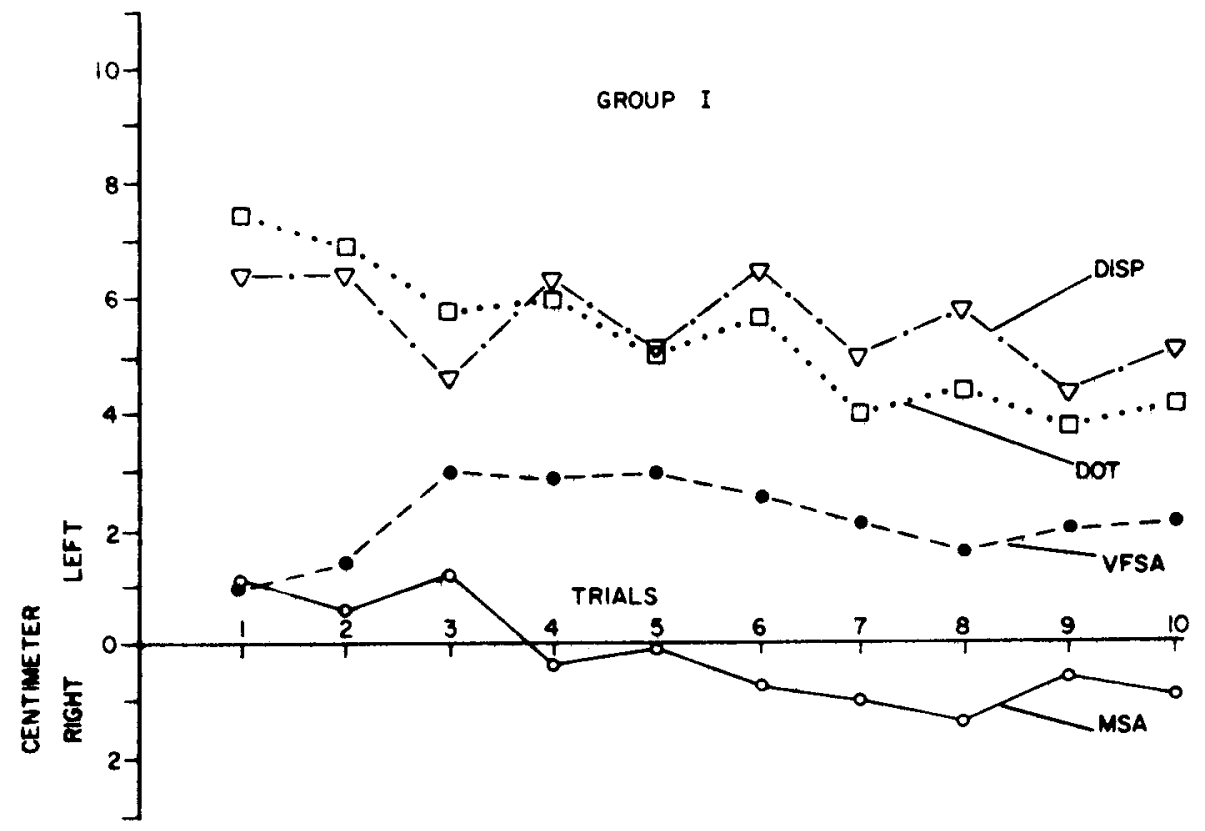

Fig. 1. Mean data for 10 exposure trials for Group 1.

the limbus was measured. The average measurements from the three or four sets of calibration photos were plotted against position of visual fixation on the horizontal row of dots. This gave an empirical relationship between $d$ and the point of fixation. The curve describing this relationship was used to determine point of fixation on each of the 10 photos taken during the exposure trials. Mclaughlin and Webster (1967) have reported that this method has a reliability of $15 \mathrm{~min}$ of arc with a standard error of $6.3 \mathrm{~min}$ of arc.

\section{RESULTS}

Group 1: Instructed to ignore any errors in visual target pointer settings

The results for the Group 1 Ss are summarized in Figs. 1 and 2. These figures show the mean pointer settings and straight-ahead eye position for the pre- and postexposure periods and the 10 adaptive exposure trials.

Analyses of the mean trial data illustrated in Fig. I reveals that straight-ahead eye position (VFSA) shifted significantly to the left over the 10 trials $(F=3.30$, df $=9 / 99, p<0.01)$. For manual straight-ahead (MSA) settings an opposite and significant shift $(F=3.31, p<0.01)$ to the right was recorded. While visual target (Dot) settings improved significantly $(F=3.95, p<0.001)$ during the 10 trials, disparity measurements (Disp) did not change $(F=1.21, p<0.05)$. "Disparity" is defined as the difference between a visual target and the adjacent straight-ahead pointer settings made on any one trial. Thus, the disparity for Trial $1(6.2 \mathrm{~cm})$ equals the visual target pointer setting (Dot) value for Trial $1(7.3 \mathrm{~cm})$ minus the straight-ahead pointer setting value for Trial $1(1.1 \mathrm{~cm})$.

Comparison of pre- and postexposure pointer settings indicates that there were no significant differences between pre- and postexposure MSA settings for pointing with either hand (see Table 1). Comparisons of pre- and postexposure Dot settings show that significant improvement followed the adaptive exposure. Improvement was significantly greater for right-handed settings suggesting an adaptive influence affecting only the right hand. Disparity measurements decreased significantly from the pre- to the postexposure periods with the amount of change for the two hands not differing significantly. Auditory target settings made with cither hand did not shift significantly.
Group 2: Instructed to compensate for and to eliminate any errors in visual target pointer settings

For Group 2, changes over trials in straight-ahead eye position (VFSA) were insignificant: An initial shift to the left was followed by a complete return to the right (Fig. 3). MSA settings appear to shift to the right in Fig. 3, but the shift was insignificant. Both Dot settings $(F=25.96, p<0.001)$ and Disp measurements $(F=7.33, p<0.001)$ registered large significant changes during the 10 trials. Dot settings became

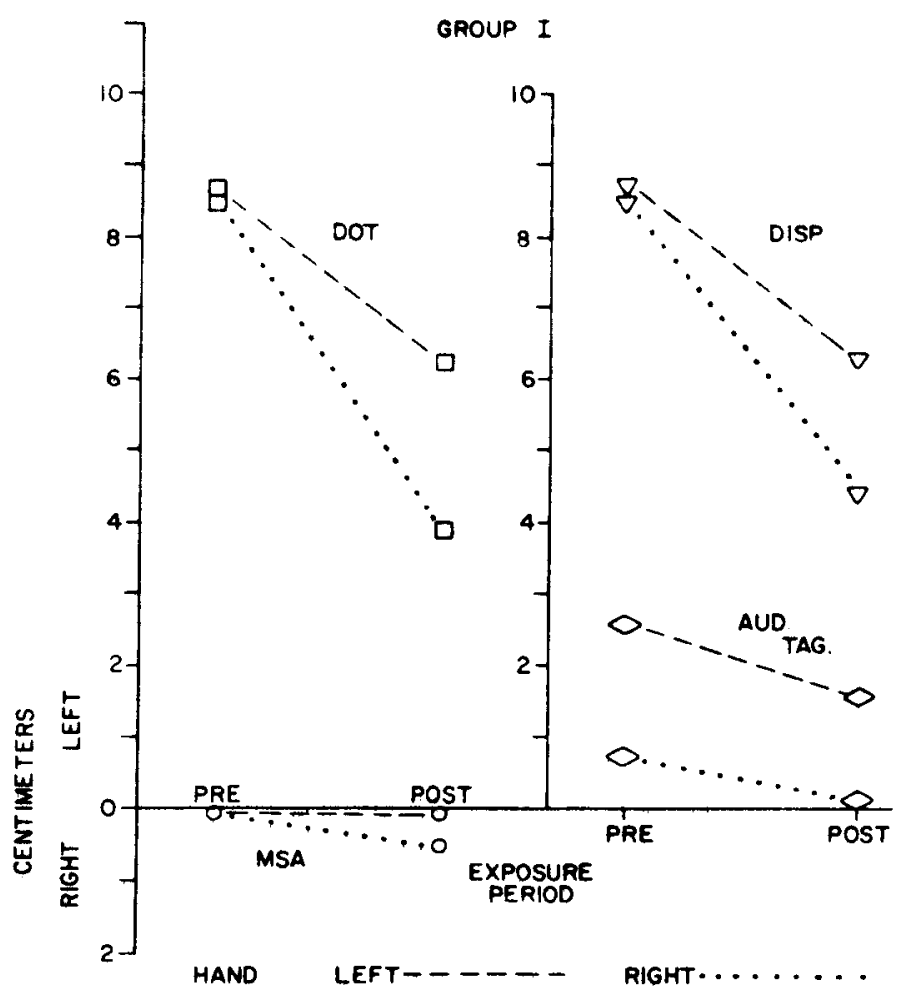

Fig. 2. Mean pre- and postexposure data for Group 1. 
Table 1

Mean Pre- and Post-Exposare Values in $\mathrm{cm}$ for Group $1(\mathrm{~N}=12)$

\begin{tabular}{|c|c|c|c|c|c|c|}
\hline Response & Hand & Pre P & Post & Difference & $S D_{\text {diff }}$ & $\mathbf{t}$ \\
\hline MSA & $\begin{array}{c}\text { Left } \\
\text { Right } \\
\text { Difference }\end{array}$ & $\begin{array}{l}-0.08 \\
-0.03 \\
-0.05\end{array}$ & $\begin{array}{rr}3 & -0.02 \\
3 & -0.53 \\
5 & 0.51\end{array}$ & $\begin{array}{r}0.06 \\
-0.50 \\
0.56\end{array}$ & $\begin{array}{l}0.55 \\
0.32 \\
0.47\end{array}$ & $\begin{array}{l}0.12 \\
1.56 \\
1.20\end{array}$ \\
\hline Dot & $\begin{array}{c}\text { Left } \\
\text { Right } \\
\text { Difference }\end{array}$ & $\begin{array}{r}8.61 \\
8.42 \\
-0.30 \\
\end{array}$ & $\begin{array}{l}6.22 \\
3.85 \\
2.37 \\
\end{array}$ & $\begin{array}{r}-2.39 \\
-4.57 \\
2.18 \\
\end{array}$ & $\begin{array}{l}0.50 \\
0.73 \\
0.66 \\
\end{array}$ & $\begin{array}{l}4.74^{* *} \\
6.26^{* *} \\
3.25^{* *}\end{array}$ \\
\hline Disp & $\begin{array}{c}\text { Left } \\
\text { Right } \\
\text { Difference }\end{array}$ & $\begin{array}{l}8.70 \\
8.45 \\
0.25\end{array}$ & $\begin{array}{l}6.24 \\
4.39 \\
1.85\end{array}$ & $\begin{array}{r}-2.46 \\
-4.06 \\
1.60\end{array}$ & $\begin{array}{l}0.86 \\
0.69 \\
0.82\end{array}$ & $\begin{array}{l}2.86^{* *} \\
5.88^{* *} \\
1.95\end{array}$ \\
\hline AT & $\begin{array}{c}\text { Left } \\
\text { Right } \\
\text { Difference }\end{array}$ & $\begin{array}{l}2.58 \\
0.76 \\
1.82\end{array}$ & $\begin{array}{l}1.57 \\
0.15 \\
1.42\end{array}$ & $\begin{array}{l}-1.01 \\
-0.61 \\
-0.40\end{array}$ & $\begin{array}{l}0.64 \\
0.73 \\
0.77\end{array}$ & $\begin{array}{l}1.60 \\
0.84 \\
0.52\end{array}$ \\
\hline
\end{tabular}

$* p<.05$

$+* p<.01$

completely veridical after four trials while Disp measurements decreased markedly.

From the pre- to the postexponre period, right-handed MSA settings shifted significantly to the right (see Fig. 4 and Table 2). No significant shift occurred for left-handed MSA settings. Although the amount of change in pointing with each hand did not differ significantly, an adaptive component specific to the right hand is suggested. Improvement in Dot settings following exposure was highly significant for pointing with either hand. Complete veridicality of Dot settings made with either hand was recorded for postexposure settings. Disparity measurements for pointer settings made with either hand decreased significantly. The amount of change for each hand did not differ significantly. Righthanded auditory target setting shifted significantly to the right. No change in left handed auditory target settings was found.

\section{INTERPRETATION OF RESULTS}

\section{Changes in Pointing Behavior}

In Group 1, two or more adaptive mechanisms were operating. As expected, "Dot" settings made with either hand improved, i.e., became more accurate, over trials. Improvement was greater for right-handed settings. Of the three adaptive mechanisms (oculomotor, motor-kinesthetic, and cognitive), oculomotor adaptation appears to have had only a minor influence in the results of Group 1 Ss. If oculomotor adaptation was responsible for the changes in pointer settings of Group $1 \mathrm{Ss}$, the shift in "Dot" settings would be accompanied by a related shift in straight-ahead eye position. As discussed by McLaughlin and Webster (1967), an appropriate change in straight-ahead eye position will cause the visual target to appear closer to straight-ahead and pointer settings will be altered accordingly. Inspection of the results for Group 1 shows, however, that the small shift in eye position which occurred from Trial 1 to Trial 10 is insufficient to explain the larger shift in "Dot" settings or the opposite shift in straight-ahead settings. Thus, while some oculomotor adaptation may have occurred, two other adaptive mechanisms appear to be important: (1) cognitive adaptation, and (2) a motor-kinesthetic factor which affected pointing with the right hand only. Presence of the first adaptive mechaniam is indicated by the improvement in visual target pointer settings made with either hand. The second factor is revealed by (1) the greater improvement in right-handed visual target settings as compared with left-handed settings, and (2) the shift to the right over trials in right-handed straight-ahead settings. Comparisons of pre- and postexposure settings reveals no such change in left-handed, straight-ahead settings. However, a third result which is necessary to support the motor-kinesthetic hypothesis was not found. There was no significant shift in auditory settings made with the right hand from the pro to the postexposure period. Any change in the motor-kinesthetic relationship of the pointing hand should cause equal changes in pointing behavior regardless of the type of target (visual, auditory, or straight-ahead). This lack of change in pointing at the auditory target means that the motor-kinesthetic hypothesis is only partially supported. In summary, the results for Group 1 are insufficient to allow acceptance of any one or a combination of the three adaptive mechanisms (oculomotor, motor-kinesthetic, or cognitive) as an explanation.

In contrast, the results for Group 2 provide strong support for a dual adaptive mechanism hypothesis involving cognitive and motor-kinesthetic adaptation. For Group 2 complete

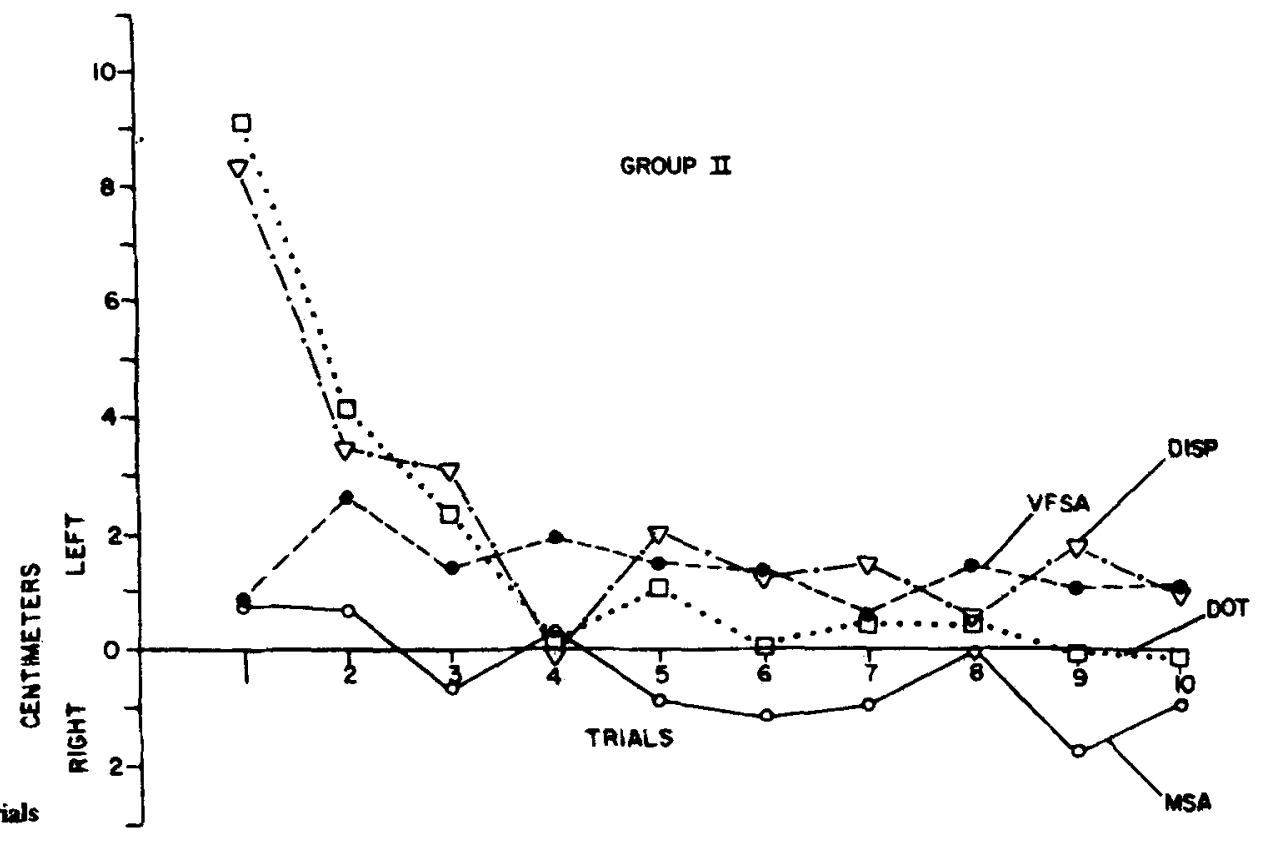

Fig. 3. Mean data for 10 exposure trials for Group 2. 


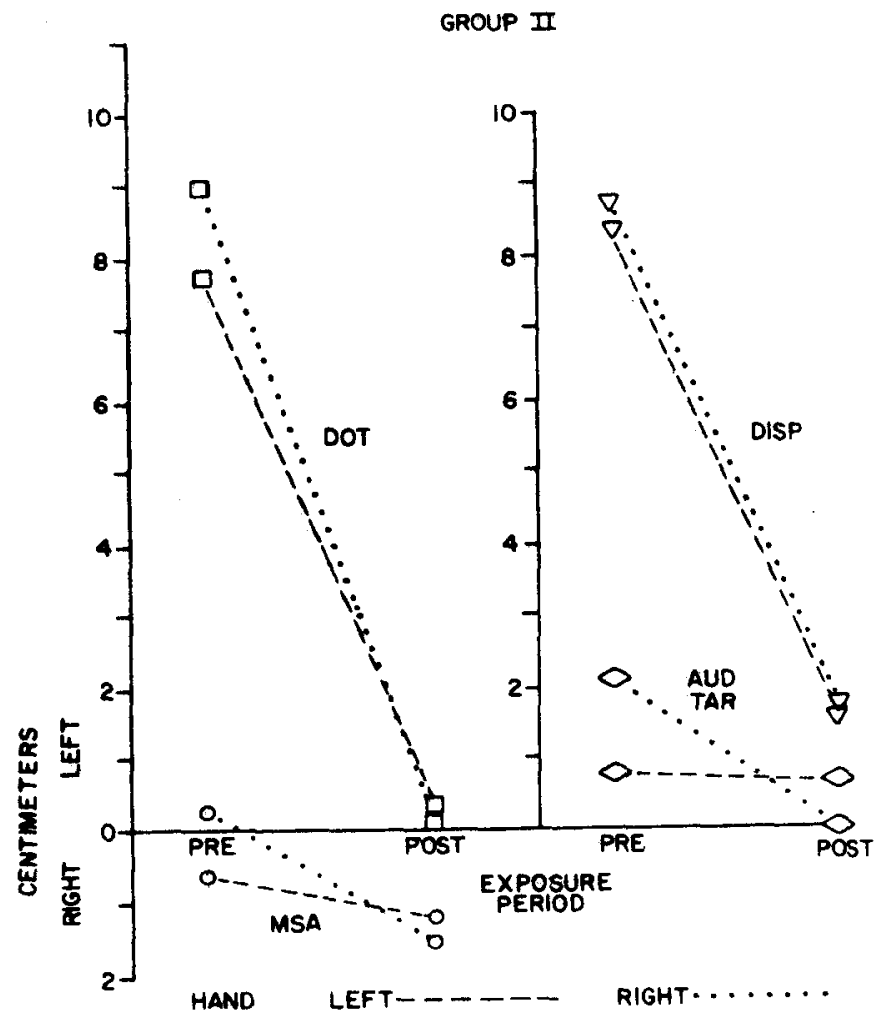

Fig. 4. Mean pre- and postexposure data for Group 2.

elimination of errors in visual target ("Dot") settings made with either hand occurred after only four exposure trials. Comparing Groups 1 and 2, the effect of instructions is obvious. Whereas $S$ s in Group 1 had the information necessary to eliminate their visual target pointing errors, they were restrained by their instructions. In Group 2 this instructional restraint was removed and complete adaptation or elimination of visual target pointing errors resulted. Because of the importance of conscious control, this form of adaptation will be called "cognitive" adaptation In addition to the rapid and complete elimination of errors in visual target settings made with either hand, right-handed straight-ahead settings shifted to the right over trials and from the pre- to the postexposure period. No similar change in left-handed. straight-ahead settings was found. This supports the motor-kinesthetic change hypothesis. In addition, and also in support of, the motor-kinesthetic change hypothesis, right-handed auditory settings shifted significantly to the right from the pre- to the postexposure period. Again, left-handed settings were apparently unaffected by the adaptive exposure. These results strongly suggest the involvement of two adaptive mechanisms. Specifically, changes in the motor-kinesthetic control loop of the right hand were accompanied by cognitive adaptation which affected pointing behavior with either hand. Both these forms of adaptation were dependent upon allowing Ss to view their prism-induced pointing errors. These errors were reduced by (1) a change in the motor-kinesthetic control loop of the pointing (right) hand, and (2) when instructions permitted, by conscious correction of visual target pointing errors.

\section{The Nature of the Adaptive Effect}

How is the information supplied to Ss when they view their prism-induced errors utilized by them? As has been shown, an apparent effect of seeing one's visual target pointing errors is a change in the motor-kinesthetic relationship of the pointing hand-in other words, a change in the judged position of the pointing hand. This change in judgment results in a change in pointing at all types of targets: visual, auditory, and straight ahead. In Groups 1 and 2 Ss were unable to see their hand and, therefore, any judgment of their hand's position must have been based on its felt position. When Ss were shown their pointing errors, their judgment of their right hand's felt position was altered resulting in more accurate visual target settings. This type of change in felt position is similar to the adaptive proprioceptive-change hypothesized by Harris.

However, in Group 2 the complete elimination of visual target pointing errors were not entirely the result of a change in the judged position of the right hand. This is shown by (1) the complete elimination of visual target pointing errors made with either hand, and (2) the comparatively small change in right-handed straight-ahead pointer settings. If motorkinesthetic changes were the only adaptive mechanism operating, the change in visual target and straight-ahead pointer settings made with the right hand would be approximately equal and there would not be a corresponding elimination of left-handed visual target pointing errors. Therefore, an additional adaptive mechanism must be involved. By viewing their pointing errors through the displacing prism, Ss were placed in a conflicting situation; the target was seen in one place but its physical position was indicated to be in another. The results of Group 2 reveal that when properly instructed, Ss were able to disregard the target's apparent visual position and were able to make their visual target pointer settings on the basis of its position as inferred from viewing the pointing errors.

\section{The Role of Oculomotor Adaptation}

The present experiment indicates that for this type of adaptive exposure, oculomotor adaptation was of minor importance. For Group 1 some oculomotor adaptation did occur but the change in eye position was insufficient to explain the larger shift in visual target pointer settings and the opposite shift in straight-ahead pointer settings. In Group 2 an initial indication of oculomotor adantation in the early exposure trials was completely eliminated during the later trials. Comparing the results of the present experiment with those of McLaughlin and Webster (1967), it is seen that oculomotor adaptation is dependent upon the type of adaptive exposure. In the McLaughlin and Webster study, significant oculomotor adaptation occurred when Ss received adaptive exposure consisting of illuminating a back panel and having Ss observe, through a prism, a moving pointer. The exposure was similar to that of the present experiment except that in the McLaughlin and Webster study, Ss were never shown their

Table 2

Mean Pre- and Post-Exposure Values in $\mathrm{cm}$ for Group $2(\mathrm{~N}=12)$

\begin{tabular}{llrrrrr}
\hline Response & Hand & Pre & Post & Difference & $\mathrm{SD}_{\text {diff }}$ & $\mathrm{t}$ \\
\hline \multirow{4}{*}{ MSA } & Left & -0.59 & -1.20 & -0.61 & 0.74 & 0.82 \\
& Right & 0.28 & -1.57 & -1.85 & 0.85 & $2.20^{*}$ \\
& Difference & -0.87 & 0.37 & 1.24 & 0.79 & 1.61 \\
\hline \multirow{4}{*}{ Dot } & Left & 7.74 & 0.37 & -7.37 & 0.85 & $8.67^{* *}$ \\
& Right & 8.98 & 0.16 & -8.82 & 0.80 & $11.02^{* *}$ \\
& Difference & -1.24 & 0.21 & 1.45 & 0.51 & $2.80^{*}$ \\
\hline \multirow{4}{*}{ Disp } & Left & 8.33 & 1.58 & -6.75 & 0.88 & $7.67^{* *}$ \\
& Right & 8.70 & 1.73 & -6.97 & 1.08 & $6.45^{* *}$ \\
& Difference & -0.37 & -0.15 & 0.22 & 0.79 & 0.27 \\
\hline \multirow{2}{*}{ AT } & Left & 0.76 & 0.65 & -0.11 & 0.55 & 0.20 \\
& Right & 2.11 & 0.00 & -2.11 & 0.39 & $5.40^{* *}$ \\
& Difference & -1.35 & 0.65 & 2.00 & 0.67 & $2.95^{* *}$ \\
\hline
\end{tabular}

${ }^{*} p<.05$

$* * p<.01$ 
prism-induced pointing errors. In contrast, Ss in the present experiment were shown their pointing errors and, in the case of Group 2, were instructed to eliminate them. In order for Group 2 Ss to eliminate their visual target pointing errors, it was necessary that they downgrade the importance of visual input. This attention to cognitive rather than visual information apparently resulted in the inhibition of oculomotor adaptation in the present experiment.

\section{CONCLUSION}

The results of the present study indicate a dependent relationship between cognitive and motor-kinesthetic adaptation. Even though Ss were unable to see their pointing hand, a change in its felt position was registered. Thus, proprioceptive adaptation as described by Harris may not depend upon seeing one's hand through prism but, rather, it may depend upon the cognitive information present when one views one's pointing errors. When given this information, which is in conflict with $S$ 's visual expectations, motor-kinesthetic and cognitive adaptation may occur and the conflict between cognitive and visual cues may be lessened.

\section{REFERENCES}

HARRIS, C. S. Adaptation to displaced vision: Visual, motor, or proprioceptive change? Science, 1963, 140,812-813.

HOWARD, I. P., CRASKE, B., \& TEMPLETON, W. B. Visuomotor adaptation to discordant exafferent stimulation. Journal of Experimental Psychology, 1965, 70, 189-191.
McLAUGHLIN, S. C., \& RIFKIN, K. I. Change in straight ahead during adaptation to prism. Psychonomic Science, 1965, 2, 107-108.

McLAUGHLIN, S. C., RIFKIN, K. I., \& WEBSTER, R. G. Oculomotor adaptation to wedge prisms with no part of the body seen. Perception \& Psychophysics, 1966, 1, 452-458.

McLAUGHLIN, S. C., \& WEBSTER, R. G. Oculomotor changes during adaptation to displaced vision. Perception \& Psychophysics, 1967, 2,
37 ,44.

von HELMHOLTZ, H. Handbuch der physiologischen Optik. Leipzig: Leopold Voss, 1867.

WEINSTEIN, S., SERSEN, E. A., FISHER, L., \& WEISINGER, M. Is reafference necessary for visual adaptation? Perceptual \& Motor Skills, $1964,18,641-648$.

\section{NOTES}

1. The present article is based on a dissertation submitted to the Department of Psychology, Tufts University, in partial fulfillment of the requirements for the Ph.D. degree. The author is deeply indebted to Samuel C. McLaughlin, Director of the Visual Perception Laboratory, Tufts University, for his exceptional patience and assistance in the execution of this study and his invaluable criticisms of the present paper. The research was supported in part by Contract MD-2714 with the U.S. Army Medical Research and Development Command, and in part by Research Grant GB-4196 from the National Science Foundation.

2. Address: Sandia Laboratories, Sandia Base, Albuquerque, New Mexico 87115 .

3. The first edition of Helmholtz' Handbuch is cited here to give an accurate date. The quotations are from J.P. C. Southall's translation of the third edition (The Optical Society of America, 1924).

(Accepted for publication October 7, 1968.) 